\title{
El sistema cannabinoide en situaciones de neuroinflamación: perspectivas terapéuticas en la esclerosis múltiple
}

\author{
L. Mestre a, F. Correa ${ }^{a}$, F. Docagne a ${ }^{\text {a D. Clemente }}{ }^{\text {a }}$, S. Ortega-Gutiérrez ${ }^{c}$, \\ A. Arévalo-Martín ${ }^{\text {b }}$ E. Molina-Holgado ${ }^{\text {b }}$,J. Borrell ${ }^{\text {a }}$, C. Guaza $^{\text {a }}$
}

\author{
CANNABINOID SYSTEM AND NEUROINFLAMMATION: \\ THERAPEUTIC PERSPECTIVES IN MULTIPLE SCLEROSIS
}

\begin{abstract}
Summary. Introduction. The endocannabinoid system consists of cannabinoid receptors, endogenous ligands and the enzymatic elements involved in their synthesis and breakdown. Aim. To report on currently held knowledge about the functioning of the system as a modulator of the neuroinflammatory processes associated with chronic diseases such as multiple sclerosis. Development. Cannabinoids are synthesised and released on demand and their production increases in times of neuroinflammation and neural damage. In this context then, their actions in the microglial cells and in the astrocytes are characterised by a lowered expression of inflammatory mediators and pro-inflammatory cytokines. Furthermore, cannabinoids can play a role as neuroprotectors by means of different types of mechanisms and, in experimental models of multiple sclerosis, they slow down the symptoms, reduce inflammation and can favour remyelination. Conclusions. The clinical use of cannabinoids or pharmacological agents that affect the endogenous cannabinoid system during inflammation of the central nervous system and in multiple sclerosis is currently under consideration and subject to debate. Detailed analysis of the results obtained over the past decade has made it possible to establish the existence of several mechanisms of action of cannabinoids in pathologies affecting the central nervous system that are accompanied by chronic inflammation. Likewise, they also clearly show that the cannabinoid system is an interesting proposal as a new therapeutic tool. [REV NEUROL 2006; 43: 541-8]
\end{abstract}

Key words. Cannabinoids. Glial cells. Immune function. Multiple sclerosis. Neuroinflammation.

\section{INTRODUCCIÓN}

El sistema cannabinoide es en la actualidad uno de los sistemas con mayor interés de estudio debido al creciente conocimiento de su implicación en la regulación de numerosos procesos fisiológicos y patológicos. La planta Cannabis sativa contiene más de 50 compuestos bioactivos, entre los que destaca el principio psicoactivo $\delta$-9-tetrahidrocannabinol (THC), aislado y caracterizado a mediados de los años sesenta [1], lo que supuso el inicio del estudio de la farmacología y la función de los cannabinoides. Otros compuestos de la planta con gran interés incluyen el cannabidiol (CBD), que es el más abundante tras el THC, y el cannabinol (CBN), que es un producto de descomposición del THC que se acumula en la planta con el transcurso del tiempo. Mientras que el THC media principalmente los efectos psicotrópicos del cannabis (alteraciones en la memoria y analgesia, entre otros efectos menos conocidos), el CBD y el CBN, que carecen de efectos psicotrópicos, son capaces de interaccionar con el endotelio vascular y el sistema inmunitario, regulando la presión sanguínea y las respuestas inflamatorias, por lo que se perfilan como compuestos prometedores en el tratamiento de patologías relacionadas con alteraciones en el sistema cardiovascular y en el sistema inmune. En el sistema nervioso central (SNC), en situaciones de neuroinflamación se ha visto que los cannabinoi-

\footnotetext{
Aceptado tras revisión externa: 07.06.06.

${ }^{a}$ Grupo de Neuroinmunología. Instituto Cajal. Centro Superior de Investigaciones Científicas. Madrid. ${ }^{b}$ Grupo de Neuroinflamación. Hospital Nacional de Parapléjicos. Toledo. ${ }^{c}$ Departamento de Química Orgánica. Facultad de Ciencias Químicas. Universidad Complutense de Madrid. Madrid, España.

Correspondencia: Dra. Carmen Guaza. Grupo de Neuroinmunología. Instituto Cajal. CSIC. Avda. Doctor Arce, 37. E-28002 Madrid. Fax: +34 915 854 754.E-mail: cgjb@cajal.csic.es

(C) 2006, REVISTA DE NEUROLOGÍA
}

des pueden afectar la patogénesis de enfermedades con un componente inmunológico, como es el caso de la esclerosis múltiple, pero también de otras patologías neurodegenerativas como la enfermedad de Alzheimer. Estas acciones beneficiosas de los cannabinoides se han tratado de explicar en base a sus propiedades antiinflamatorias y neuroprotectoras. Por ello, determinar las dianas celulares, los modos de acción y los mecanismos de señalización del sistema cannabinoide es crucial para entender la fisiología y farmacología de estos mediadores lipídicos y poder desarrollar nuevas estrategias terapéuticas.

\section{SISTEMA ENDOCANNABINOIDE}

El enorme interés en el estudio del sistema cannabinoide durante los últimos años se debe sin duda al descubrimiento del sistema endógeno. El sistema endógeno cannabinoide (SEC) está constituido por los receptores cannabinoides, sus ligandos endógenos y los enzimas que los producen e inactivan [2] (Fig. 1). Los agonistas cannabinoides exógenos (procedentes de la planta o sintéticos) y endógenos activan diferentes subtipos de receptores cannabinoides (CB): un primer grupo está constituido por los receptores CB1 y CB2, bien conocidos, caracterizados y clonados, y un segundo grupo incluiría dos tipos de receptores, caracterizados farmacológicamente pero sin clonar por el momento, que consisten en un nuevo receptor cannabinoide en el endotelio vascular, sensible al análogo del constituyente no psicotrópico del cannabis -el CBD-, denominado receptor anormal cannabidiol (Abn-CBD), y el receptor central, sensible al cannabinoide endógeno anandamida (AEA) y al agonista sintético no selectivo WIN 55,212-2, denominado receptor CB-WIN $[3,4]$. Algunas evidencias señalan además que el receptor vaniloide de tipo I (TRPVI), que es un canal iónico de potencial transitorio implicado en procesos de nocicepción y de inflama- 
ción neurogénica, puede ser diana del endocannabinoide AEA [5]. En la actualidad existen aún numerosos aspectos pendientes de clarificar y no se puede descartar la existencia de nuevos elementos en el SEC. La disponibilidad de antagonistas selectivos de los receptores $\mathrm{CB} 1$ y $\mathrm{CB} 2$ ha sido de gran ayuda en términos de poder establecer el papel de los endocannabinoides en diversos procesos fisiológicos.

El receptor CB1 se expresa abundantemente en el cerebro, principalmente en neuronas, y fuera del SNC se expresa en un nivel mucho menor [6]; es un receptor muy bien conservado desde el punto de vista evolutivo. Tiene una localización neuronal predominantemente presináptica en áreas del cerebro relacionadas con los efectos conductuales y farmacológicos asignados a los cannabinoides. El receptor CB1 también se expresa en oligodendrocitos y en progenitores, especialmente durante el desarrollo, por lo que se piensa en su implicación en la formación de mielina [7,8]; su presencia en progenitores neurales se ha relacionado con un papel en la neurogénesis [9]. Recientes estudios señalan que el SEC regula la diferenciación de progenitores neurales en el cerebro adulto promoviendo la diferenciación astroglial [10]. En cuanto a la localización periférica del receptor CB1 se sabe que está presente en el endotelio vascular, los nervios periféricos, los testículos y células del sistema inmune. El receptor $\mathrm{CB} 2$, denominado también receptor periférico, se encuentra mayoritariamente en el sistema inmune [11], aun cuando la existencia en el SNC no se puede descartar. De hecho, en situaciones patológicas como la enfermedad de Alzheimer, el receptor CB2 se expresa en microglía asociada a placas neuríticas $[12,13] \mathrm{y}$, recientemente, se ha descrito su presencia en determinadas poblaciones neuronales del tronco cerebral [14]. Ambos tipos de receptores pertenecen a la superfamilia de receptores acoplados a proteínas $\mathrm{G}$ y presentan la estructura característica de siete hélices transmembrana. En particular, entre los efectos asociados a su activación mediados por proteínas $\mathrm{G}$ pertenecientes al tipo $\mathrm{G}_{\mathrm{i} / \mathrm{o}}$, destacan la inhibición de la adenilato ciclasa y la regulación de canales iónicos (inhibición de los canales de calcio dependientes de voltaje tipo L, N y P/Q, y regulación de los canales rectificadores de potasio, $\mathrm{K}_{\mathrm{ir}}$ ) [15]. Las respuestas de agonistas cannabinoides mediadas a través de la interacción con los receptores CB1 y CB2 son sensibles a la toxina pertúsica, lo que sugiere su acoplamiento a proteínas $\mathrm{G}$ inhibitorias (Gi). Sin embargo, ciertos estudios sugieren la activación de proteínas $\mathrm{G}$ estimuladoras (Gs). La obtención de ratones deficientes del receptor $\mathrm{CB} 1, \mathrm{CB} 2$ o dobles deficientes han permitido defender la existencia de nuevos subtipos de receptores al observarse que algunos agonistas como el compuesto WIN
55212,2 continuaban inhibiendo la liberación de glutamato en el hipocampo de ratones deficientes de receptores CB1 [16].

En cuanto a la historia del descubrimiento de los ligandos endógenos, en 1992 el grupo de Mechoulam [17] identificó y caracterizó el primer endocannabinoide, la araquidonil etanolamida, denominado anandamida (AEA). Este lípido se sintetiza en demanda a partir de los fosfolípidos de la membrana plasmática, mediante un proceso dependiente de calcio que es necesario para la activación de los sistemas enzimáticos implicados en su biosíntesis [18]. En 1995 se identificó el segundo endocannabinoide, 2-araquidonil glicerol (2-AG), que también requiere calcio para su síntesis y es más abundante que la AEA en el SNC [19]. Más recientemente se añadieron dos nuevos compuestos, un análogo del 2-AG, el noladin éter, y la virodhamina, los cuales representan el tercer y cuarto endocannabinoide, pero no está clara su presencia en el cerebro. Existen además diferentes lípidos derivados de ácidos grasos y relacionados estructuralmente con AEA y 2-AG que en algunos casos también pueden interaccionar con receptores cannabinoides o realizar acciones independientes de la activación del receptor CB1 o CB2. En el caso de la palmitoil etanolamina (PEA), se la considera un subtipo particular de endocannabinoide, ya que se produce e inactiva en las células y ejerce acciones biológicas relacionadas con la inhibición de la inflamación, si bien no está claro a través de qué tipo de receptor cannabinoide actúa. Debe tenerse en cuenta que existen además compuestos relacionados estructuralmente con los endocannabinoides, como algunos derivados del ácido araquidónico; es el caso de la L-araquidonoil serina (ARA), que ejerce acciones diversas en el sistema vascular [20]. La acción biológica de los endocannabinoides finaliza mediante un sistema de inactivación que incluye mecanismos de desensibilización e internalización del receptor cannabinoide y me- 


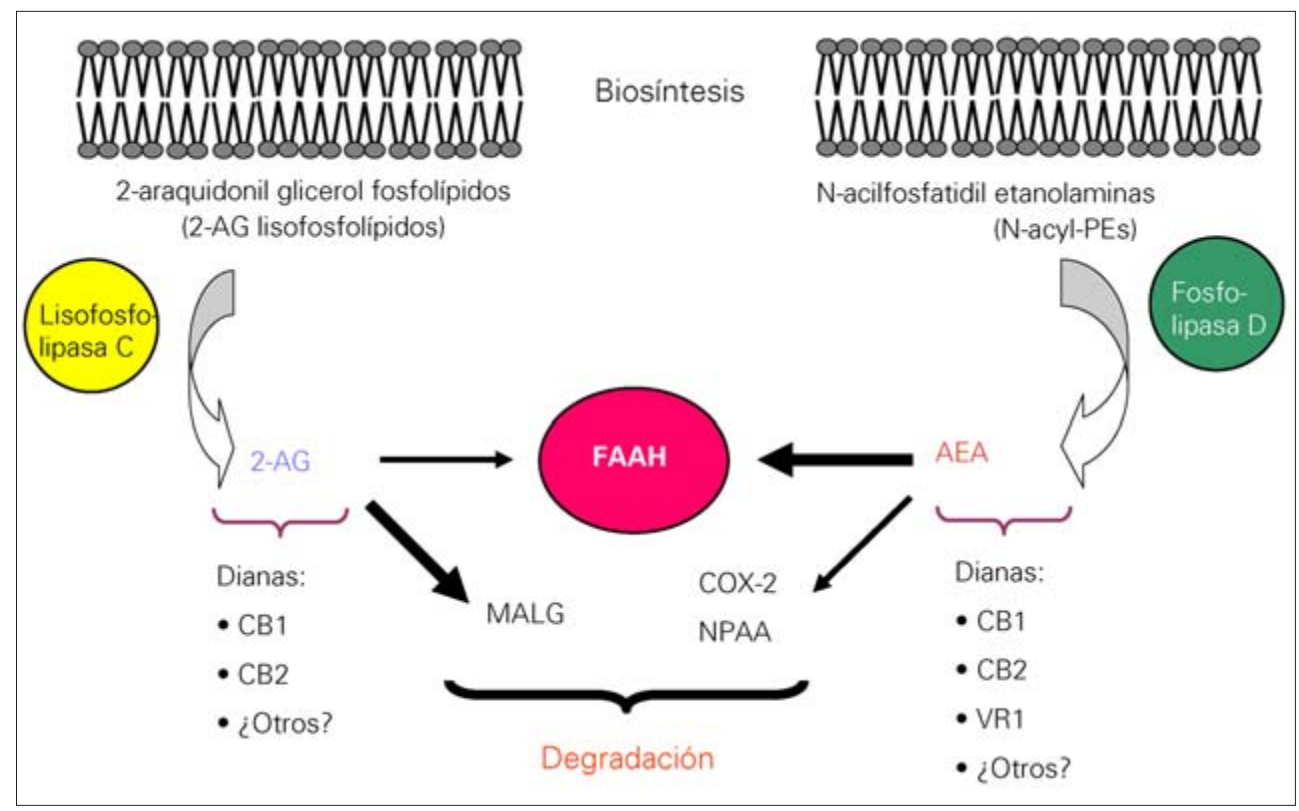

Figura 2. Vías sintéticas y degradativas de los dos endocannabinoides más representativos: anandamida (AEA) y 2-araquidonil glicerol (2-AG). La AEA tiene el mismo mecanismo de biosíntesis que el resto de las $\mathrm{N}$-aciletanolaminas. En una primera etapa, un enzima con actividad transacilasa dependiente de calcio cataliza la transferencia de un resto de ácido graso de un fosfolípido al grupo amino de la etanolamina, formando N-acilfosfatidiletanolamina; en una segunda etapa, ésta sufre una hidrólisis por una fosfolipasa D recientemente caracterizada (NAPE fosfolipasa D). El 2-AG se sintetiza a partir de fosfatidil inositol, que es sustrato de una fosfolipasa C, generando 1,2-diacilglicerol, y de éste, tras hidrólisis por una lipasa, se produce 2-AG. La degradación de la AEA implica principalmente el enzima amidohidrolasa de los ácidos grasos (FAAH), pero puede ser también sustrato de ciclooxigenasa-2 (COX-2) y de NPAA, mientras que el 2-AG es degradado mayoritariamente por una lipasa de monoglicéridos (MALG) recientemente caracterizada.

diante la eliminación de los endocannabinoides del medio extracelular por un proceso de transporte-recaptación e hidrólisis intracelular.

El fenómeno de cómo ocurre el proceso de recaptación de endocannabinoides es actualmente materia de debate. Existen distintas hipótesis, pero últimamente está siendo objeto de creciente atención la que atribuye el fenómeno de transporte a través de la membrana celular a un simple efecto de difusión dirigido por la acción de los enzimas de degradación intracelular [2]. En la actualidad se está intentando establecer si existe o no una proteína con función transportadora de la AEA o cuál es realmente la naturaleza de este transporte. En el interior celular, la AEA se hidroliza en ácido araquidónico y etanolamina mediante el enzima amidohidrolasa de los ácidos grasos (FAAH) [21]. En el caso del cannabinoide 2-AG, se sabe que es mayoritariamente hidrolizado por la acción de una lipasa de monoglicéridos (MGL) recientemente caracterizada [22]. Además, se ha demostrado que la AEA puede ser sustrato del enzima ciclooxigenasa-2 (COX-2) y dar lugar a compuestos denominados prostamidas [23], con acciones en parte relacionadas con las prostaglandinas, aunque no se dispone de evidencias claras de sus mecanismos de acción (Fig. 2). El desarrollo durante los últimos años de compuestos sintéticos cada vez más potentes, selectivos y con acciones farmacológicas definidas capaces de actuar en las distintas proteínas que constituyen el SEC, ha incidido de manera notable en la consideración de éste como diana potencial terapéutica en patologías muy diversas debido a su amplio espectro de acción.

En resumen, la familia de los cannabinoides endógenos se compone de lípidos estructuralmente relacionados, pero con pro- piedades farmacológicas diferentes y que interaccionan con receptores cannabinoides. Este sistema tiene un amplio espectro de influencia en circuitos neuronales excitatorios e inhibitorios, y está implicado en la regulación de un buen número de funciones fisiológicas. La posibilidad de actuar como mensajeros retrógrados al generarse de manera dependiente de calcio en los terminales postsinápticos y poder difundir a la terminal presináptica e inhibir la liberación de neurotransmisores, refuerza la capacidad de interacción de los endocannabinoides con diferentes sistemas de neurotransmisión [24,25]. La acción de los cannabinoides en el SNC se extiende a las células gliales, que al menos en el caso de los astrocitos y la microglía están provistas de la maquinaria necesaria no sólo para responder, sino también para producir e inactivar endocannabinoides [13]. En condiciones neuropatológicas en las que la barrera hematoencefálica está alterada, los leucocitos acceden al cerebro y participan en las respuestas inflamatorias, estableciéndose una red de comunicación entre células intrínsecas del cerebro y las células inmunitarias, que también son diana de las acciones de los cannabinoides, lo que puede estar en la base de los beneficios de los cuales se ha venido informando en relación con los cannabinoides y la esclerosis múltiple.

\section{SISTEMA CANNABINOIDE Y NEUROINFLAMACIÓN: CÉLULAS GLIALES}

En situaciones de neuroinflamación se produce un cambio dinámico y complejo en el fenotipo de las células gliales. Unas de las primeras células en responder a la inflamación son las células microgliales que retraen sus procesos y migran hacia el sitio del daño, donde liberan citocinas proinflamatorias como la interleucina (IL) $1 \beta$, el factor de necrosis tumoral alfa (TNF- $\alpha$ ) y la IL-6, lo que estimula el reclutamiento y activación de los leucocitos. De esta manera, la microglía regula la iniciación y progresión de la respuesta inmune intracerebral. Las células microgliales activadas expresan el receptor cannabinoide CB2 y el nivel de expresión depende del grado de activación de éstas, como ocurre también en los macrófagos y en las células dendríticas [26]. Además, la microglía produce y libera endocannabinoides en una proporción unas 20 veces superior a lo observado en neuronas y astrocitos, proporción que varía dependiendo del subtipo de endocannabinoide y del estímulo [13,27]. El sistema cannabinoide participa en la regulación de la función microglial a diferentes niveles: por un lado, bloqueando la liberación de agentes citotóxicos y citocinas proinflamatorias, y por otro, fa- 
voreciendo la migración y regulando la proliferación celular, todo ello encaminado -parece ser- a limitar el proceso inflamatorio y prevenir la propagación y el daño celular [2730]. Los astrocitos expresan receptores $\mathrm{CB} 1$, aunque hay cierta controversia al respecto, y también son capaces de producir endocannabinoides en respuesta a ionóforos de calcio, endotelina o ATP [31]. El tipo de endocannabinoide producido varía según el estímulo, en particular ATP aumenta selectivamente la producción de 2-AG, de manera que un determinado estímulo puede favorecer la síntesis de un endocannabinoide específico. Otro aspecto importante a considerar es que los astrocitos expresan el enzima FAAH, implicado en la degradación de AEA y de 2-AG, mientras que la microglía expresa preferentemente MGL, implicada de manera especial en la degradación de 2-AG [13]. En el contexto de la neuroinflamación, nuestro grupo describió en 1997 que la AEA inhibía la producción de óxido nítrico y de TNF- $\alpha$ en astrocitos infectados con el virus de Theiler [32]. En estudios posteriores verificamos la expresión del receptor CB1 en la astroglía -al menos en el caso de la inhibición del enzima óxido nítrico sintasa tipo II, los efectos eran dependientes del receptor CB1-, así como el efecto potenciador de la AEA sobre la liberación de IL-6 [33-35]. En otros trabajos se mostraba que parte de los efectos antiinflamatorios y neuroprotectores de los cannabinoides eran mediados por la inducción del antagonista del receptor de IL-1 (IL-1ra) en la microglía y los astrocitos [36]. Esta última observación resulta de especial interés puesto que el IL-1ra limita las acciones de la IL-1 $\beta$, una de las citocinas que primero responde a la inflamación y que puede tener un efecto dual de reparación o de propagación del daño en el caso de que su producción sea excesiva y continuada. Por otro lado, algunos datos apuntan a una acción del sistema cannabinoide sobre los mecanismos de inmunidad innata y adquirida, por ejemplo en el caso de la encefalomielitis murina por infección con el virus de Theiler a través de interacciones con la microglía, célula intrínseca del cerebro con capacidad de presentar antígenos. Un hecho significativo a este respecto es el efecto inhibitorio inducido por agonistas cannabinoides del receptor CB2, así como por el endocannabinoide AEA, sobre la producción de citocinas de la familia de la IL-12 en los macrófagos y en la microglía [37,38]. Este grupo de citocinas heterodiméricas (IL-12, IL-23, IL-27), generadas fundamentalmente por células de la estirpe mieloide, desempeñan un papel clave en la iniciación, desarrollo y establecimiento de respuestas de inmunidad adquirida, ya que interaccionan de manera secuencial con linfocitos T vírgenes, linfocitos Th1 efectores y linfocitos Th1 de memoria, favoreciendo su expansión clonal (Fig. 3). Alteraciones en la regulación sobre la producción de esta familia de citocinas se han relacionado con el desarrollo de procesos de autoinmunidad e inflamación crónica, como es el caso de la esclerosis múltiple, entre otras patologías.

\section{SISTEMA CANNABINOIDE Y ESCLEROSIS MÚLTIPLE}

La esclerosis múltiple es la enfermedad crónica inflamatoria del SNC más frecuente en jóvenes adultos y una de causas más comunes de discapacidad laboral. Es una enfermedad de etiología desconocida mediada por el sistema inmune y que cursa con desmielinización. En relación con la evidencia clínica existen ciertas anécdotas sobre los posibles beneficios que el consumo de hachís puede tener en pacientes con esclerosis múltiple. Así, los resultados de cuestionarios enviados a pacientes muestran en general una ligera mejoría de algunos de los síntomas característicos de esta enfermedad (espasticidad, dolor crónico, temblor) después del consumo de cannabis. En la actualidad, hay en marcha ensayos clínicos en diferentes países y se ha comercializado en Canadá un preparado de la planta (Sativex ${ }^{\circledR}$, GW Pharmaceuticals). En el Reino Unido se ha llevado a cabo un ensayo 


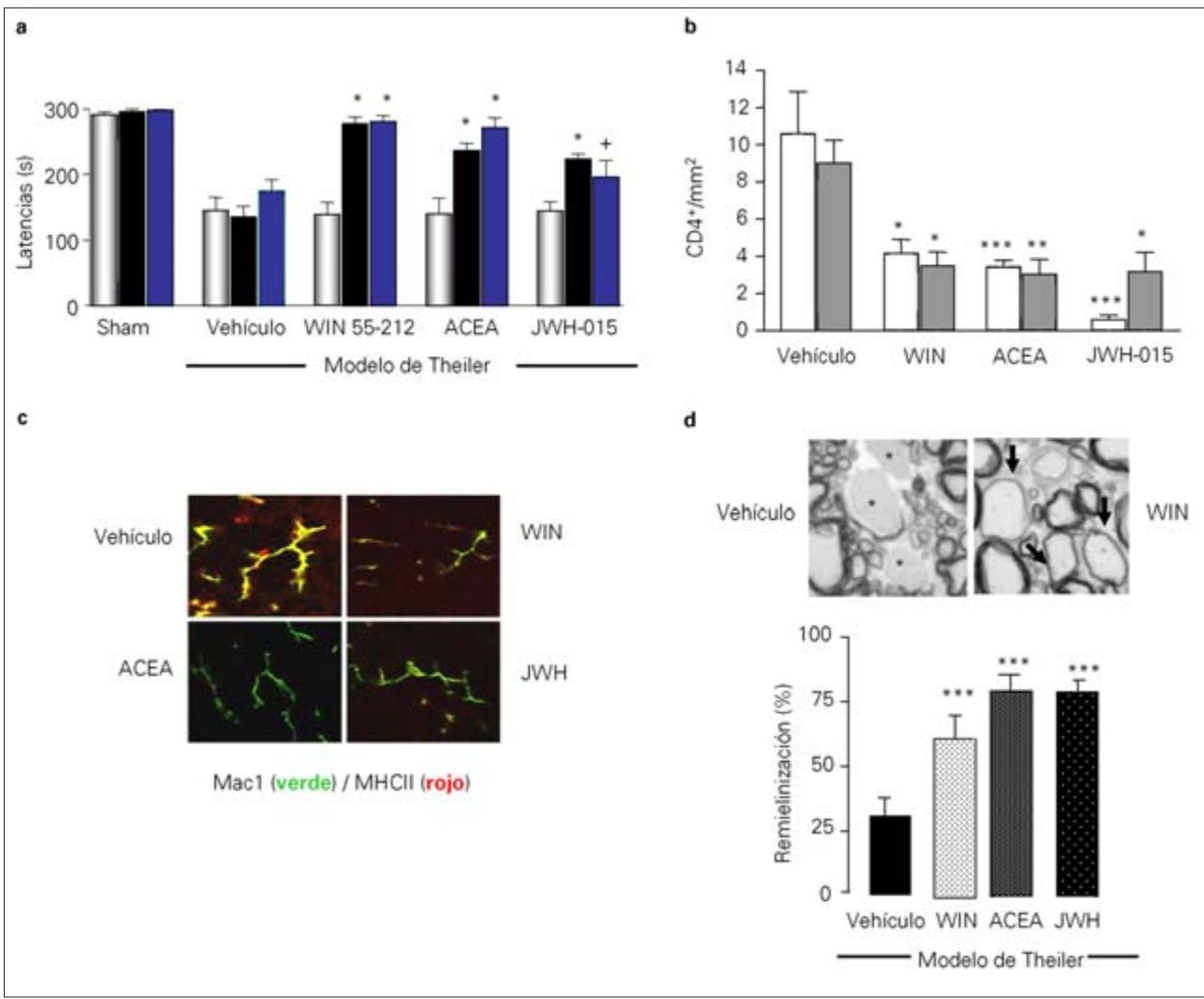

Figura 4. Efectos de los cannabinoides en el modelo vírico de esclerosis múltiple: encefalomielitis murina por infección con el virus de Theiler (TMEV). Acciones de diferentes agonistas cannabinoides -no selectivo (WIN), selectivo CB1 (ACEA) y selectivo CB2 (JWH-015)- administrados durante 10 días consecutivos a ratones infectados con el virus de Theiler (modelo de esclerosis múltiple) una vez establecida la sintomatología. a) Valoración de la función motora mediante la ejecución del test de rotarod: las deficiencias en la ejecución observadas en ratones con TMEV y que reciben vehículo se restauran tras el tratamiento con cannabinoides un día (histograma negro) o 25 días después de finalizado el tratamiento (histograma gris oscuro); b) El tratamiento con cannabinoides reduce el número de infiltrados CD4+; c) También disminuye la expresión de moléculas del MHC de clase II manteniéndose los efectos al menos 25 días después de cesado el tratamiento; d) El mismo tratamiento promueve la remielinización valorada en secciones de médula espinal a los 25 días del tratamiento (adaptado de [43]).

que merece destacarse porque representa el primer estudio de ensayo clínico a gran escala (Medical Research Council). Los resultados ya publicados de este estudio muestran una cierta expectativa de mejoría en los pacientes (espasticidad, mejora en el inicio del movimiento y dolor) tras el tratamiento con un compuesto cannabinoide [39]. Quizás lo más significativo de este ensayo es la constatación de una mejor evolución de la enfermedad tras el seguimiento de los pacientes un año después de cesado el tratamiento [40].

En modelos experimentales como la encefalitis alérgica experimental crónica en su modalidad recurrente remitente (CREAE) se han realizado algunos estudios, siendo el más significativo el trabajo Baker et al [41], que abrió ciertas expectativas al mostrar que los cannabinoides mejoraban la espasticidad muscular mediante mecanismos mediados por receptores CB1 y CB2. En otro trabajo del mismo grupo se observaba que se producía una activación del tono endocannabinoide en la médula espinal de los ratones afectados en relación con la espasticidad [42]. Parece ser, por tanto, que los efectos beneficiosos de los cannabinoides sobre la esclerosis múltiple podrían relacionarse en parte con sus acciones sobre vías motoras y también vías nociceptivas. Sin embargo, teniendo en cuenta el componente inmunológico de la esclerosis múltiple, existen otros me- canismos alternativos que pueden relacionarse con las acciones inmunomoduladoras y antiinflamatorias de los cannabinoides. En el modelo de Theiler, que contempla la posible etiología vírica de la esclerosis múltiple y reproduce las características de esta enfermedad en su fase progresiva, nuestro grupo mostró que la administración subcrónica de cannabinoides sintéticos (agonistas $\mathrm{CB} 1$ o $\mathrm{CB} 2$ ) a ratones que presentaban una clara deficiencia neurológica conducía a una recuperación funcional motora, junto con una drástica reducción en la reactividad inflamatoria en la médula espinal, y a una potenciación de la remielinización (Fig. 4) [43]. En otro trabajo simultáneo [44] se describía, también en el modelo de Theiler, una atenuación de la gravedad de la sintomatología tras el tratamiento con un agonista cannabinoide no selectivo, junto con alteraciones inmunológicas como la disminución en la expresión de citocinas proinflamatorias en la médula espinal de los animales infectados. Estudios posteriores encaminados a valorar qué ocurría si se aumentaba el tono endógeno cannabinoide tras la manipulación farmacológica de éste mediante la utilización de inhibidores de la recaptación, mostraron efectos beneficiosos en el modelo de Theiler. Estos efectos incluían una mejora en la función motora y una disminución en el grado de inflamación y en la producción de mediadores inflamatorios en los astrocitos y en la microglía $[35,38,45]$. También en otros modelos experimentales de esclerosis múltiple se observaban resultados similares tras la administración de inhibidores de la recaptación o el metabolismo de los cannabinoides endógenos [46]. En estos estudios, y en otros, se ha valorado el estatus del sistema cannabinoide en modelos animales de esclerosis múltiple [38,46,47], y en tejido post mortem de pacientes diagnosticados de esclerosis múltiple en diferentes estadios de evolución de la enfermedad [48]. En este último trabajo se observa un aumento en la concentración de AEA en las lesiones activas casi cuatro veces superior a lo observado en muestras de individuos control [48]. Estas observaciones estarían de acuerdo con el concepto de que el SEC se activaría en situaciones patológicas de inflamación con objeto, tal vez, de proteger o reparar el daño limitando la respuesta inmunológica, lo que representaría un nuevo mecanismo de comunicación neuroinmune.

Si se tiene en cuenta además el componente neurodegenerativo de la esclerosis múltiple y la importancia del daño axonal 
[49] como causa primaria de la discapacidad funcional en esta enfermedad, es interesante señalar que ratones deficientes del receptor CB1 muestran un aumento significativo del daño axonal en el modelo de CREAE [50]. Por otra parte, algunos datos apuntan a una participación de mecanismos excitotóxicos en el daño axonal en modelos experimentales de esclerosis múltiple y existen evidencias que muestran que los receptores cannabinoides interfieren con la excitoxicidad mediante la inhibición de la liberación de glutamato [51], de manera que se están comenzando a esclarecer los múltiples mecanismos y las vías de actuación de los receptores cannabinoides en relación con la neuroinflamación y las patologías neurodegenerativas, como es el caso de la esclerosis múltiple. Sin embargo, todavía se desconocen aspectos fundamentales del ámbito celular y molecular; por ejemplo, nuestro grupo describió la expresión de receptores cannabinoides en progenitores de oligodendrocitos y la inducción de supervivencia por diferentes agonistas cannabinoides en situaciones adversas como falta de soporte trófico o neuroinflamación [8], pero por el momento desconocemos si estas acciones observadas in vitro están implicadas en el aumento de la remielinización que observamos in vivo en el modelo de encefalomielitis murina por infección con el virus de Theiler [43].

En situaciones crónicas inflamatorias, en enfermedades autoinmunes o en trastornos en los que existe una hiperactivación inmunológica, los efectos beneficiosos de los cannabinoides pueden relacionarse con sus propiedades inmunomoduladoras. Es el caso de la artritis reumatoide, en que la administración del compuesto natural cannabinol mejora considerablemente la sintomatología en un modelo experimental de esta enfermedad [52], o los casos comentados anteriormente en el modelo CREAE o en el propio modelo vírico de Theiler. Los cannabinoides afectan a un importante número de funciones inmunitarias $\mathrm{y}$, así, pueden modular funciones de linfocitos $\mathrm{T}$ (como proliferación y citotoxicidad), la formación de anticuerpos por linfocitos $\mathrm{B}$, o afectar a la producción de algunas citocinas, ya que disminuyen las denominadas proinflamatorias (IL-2, IL-12, interferón- $\gamma$ ) y aumentan los niveles de antiinflamatorias (IL-4, IL-10) [53]. Sin embargo la caracterización de estas acciones, los receptores implicados y las vías de señalización están poco estu- diadas por el momento y es un tema de alto interés en la actualidad. Otro aspecto importante a tener en cuenta es el papel que podría desempeñar el SEC en la permeabilidad de la barrera hematoencefálica y en la producción de moléculas de adhesión celular por el endotelio cerebral, que tiene un especial interés en el caso de la esclerosis múltiple. Hasta la fecha, dos trabajos han mostrado una disminución de infiltrados leucocitarios en modelos experimentales de esclerosis múltiple $[43,54]$ tras el tratamiento con cannabinoides.

\section{CONCLUSIONES}

Teniendo en cuenta que el primer receptor cannabinoide se clonó en 1990 y el primer ligando endógeno en 1992, se ha realizado un extraordinario progreso en el conocimiento del SEC. En particular, en relación con la neuroinflamación, es de destacar su papel inmunomodulador-protector. El uso clínico de cannabinoides o agentes farmacológicos que inciden en el SEC durante la inflamación del SNC y en la esclerosis múltiple está actualmente sometido a consideración y debate. El análisis de los resultados obtenidos en la última década procedentes de investigación básica ha permitido establecer que son múltiples los mecanismos de actuación de los cannabinoides y en diferentes dianas celulares los que podrían sustentar las acciones beneficiosas del SEC en las patologías del SNC con inflamación crónica, en particular en el caso de la esclerosis múltiple. Por un lado, podemos considerar los aspectos paliativos de mejora de la sintomatología que englobarían, en parte, acciones relacionadas con la espasticidad muscular y la nocicepción; también tendríamos que considerar los aspectos de reparación y recuperación funcional que se relacionarían con la participación del sistema cannabinoide en la regulación del proceso inmunoinflamatorio, y en los mecanismos de neuroprotección y de remielinización. Se necesitará la convergencia de una investigación multidisciplinaria para comprender la función fisiológica y fisiopatológica de este sistema de señalización lipídica de reciente reconocimiento para establecer si los cannabinoides constituyen una nueva herramienta terapéutica con ventajas sobre otros fármacos para el tratamiento de la esclerosis múltiple.

\section{BIBLIOGRAFÍA}

1. Gaoni Y, Mechoulam R. Isolation, structure and partial synthesis of an active constituent of hashish. J Am Chem Soc 1964; 86: 1646-7.

2. Lambert MD, Fowler CJ. The endocannabinoid system: drug targets, lead compounds, and potential therapeutic applications. J Med Chem 2005; 48: 5059-87.

3. Offértaler L, Mo F, Bátkai S, Liu J, Begg M, Razdan RK, et al. Selective ligands and cellular effectors of a $\mathrm{G}$ protein-coupled endothelial cannabinoid receptor. Mol Pharmacol 2003; 63: 699-705.

4. Di Marzo V, Breivogel CS, Tao Q, Bridgen DT, Razdan RK, Zimmer AM, et al. Levels, metabolism and pharmacological activity of anandamide in CB1, cannabinoid receptor knock-out mice: evidence for non $\mathrm{CB} 1$, nor $\mathrm{CB} 2$ receptor mediated actions of anandamide in mouse brain. J Neurochem 2000; 75: 2434-44.

5. Di Marzo V, Blumberg PM, Szallasi A. Endovanilloid signalling in pain. Curr Opin Neurobiol 2002; 12: 372-9.

6. Matsuda LA, Lolait SJ, Browstein MJ, Young AC, Bonner TI. Structure of a cannabinoid receptor and functional expression of the cloned cDNA. Nature 1990; 346: 561-4.

7. Fernández-Ruiz J, Gómez M, Hernández M, De Miguel R, Ramos JA. Cannabinoids and gene expression during brain development. Neurotox Res 2004; 6: 389-91.

8. Molina-Holgado E, Arévalo-Martín A, Vela JM, Almazán G, MolinaHolgado F, Borrell J, et al. Cannabinoids promote oligodendrocyte progenitor survival: involvement of cannabinoid receptors and phosphatidylinositol-3 kinase/Akt signalling J Neurosci 2002; 22: 9742-53.
9. Aguado T, Monory K, Palazuelos J, Stella N, Cravat B, Lutz B, et al. The endocannabinoid system drives neural progenitors proliferation. FASEB J 2005; 19: 1704-6.

10. Aguado T, Palazuelos J, Monory K, Stella N, Cravat B, Lutz B, et al. The endocannabinoid system promotes astroglial differentiation by acting on neural progenitors cells. J Neurosci 2006; 26: 1551-61.

11. Galiegue S, Mary S, Marchand J, Dussossoy D, Carriere D, et al. Expression of central and peripheral cannabinoid receptors in human immune tissues and leukocyte subpopulations. Eur J Biochem 1995; 232: 54-61.

12. Benito C, Núñez E, Tolón RM, Carrier EJ, Rábano A, Hillard CJ, et al. Cannabinoid CB2 receptors and fatty cid amide hydrolase are selectively overexpressed in neuritis plaques-associated glia in Alzheimer disease. J Neurosci 2003; 23: 11136-41.

13. Stella N. Cannabinoid signalling in glial cells. Glia 2004; 48: 267-77.

14. Van Sickle MD, Duncan M, Kingsley PJ, Mouihate A, Urbani P, Mackie $\mathrm{M}$, et al. Identification and functional characterization of brainstem cannabinoid CB2 receptors. Science 2005; 310: 329-32.

15. Howlett AC. Pharmacology of cannabinoid receptors. Ann Rev Pharmacol Toxicol 1995; 273: 734-43.

16. Hájos N, Ledent C, Freund TF. Novel cannabinoid-sensitive receptors mediates inhibition of glutamatergic synaptic transmission in the hippocampus. Neuroscience 2001; 106: 1-4.

17. Devane WA, Hanus L, Breuer A, Pertwee RG, Stevenson LA, Griffin $\mathrm{G}$, et al. Isolation and structure of a brain constituent that binds to the cannabinoid receptor. Science 1992; 258: 1946-9. 
18. Di Marzo V, Fontana A, Cadas H, Schinelli S, Cimino G, Schwartz JC, et al. Formation and inactivation of endogenous cannabinoid anandamide in central neurons. Nature 1994; 372: 689-91.

19. Mechoulam R, Ben-Shabat S, Hanus L, Ligumsky M, Kaminski NE, Schatz AR, et al. Identification of an endogenous 2-monoglyceride, present in canine gut, that binds to cannabinoid receptors. Biochem Pharmacol 1995; 50: 83-90.

20. Milman G, Maor Y, Abu-Lafi, S, Horowitz, M, Gallily R, Batkai, S, et al. $\mathrm{N}$ arachidonoyl-L-serine, an endocannabinoid like brain constituent with vasodilatory properties. Proc Natl Aca Sci USA 2006; 103: 2428-33.

21. Cravatt BF, Giang DK, Mayfield SP, Boger DL, Lerner RA, Gilula NB. Molecular characterization of an enzyme that degrades neuromodulatory fatty-acid amides. Nature 1996; 384: 83-7.

22. De Petrocellis L, Cascio MG, Di Marzo V. The endocannabinoid system: a general view and latest additions. Br J Pharmacol 2004; 141: 765-74.

23. Ross RA, Craib SJ, Stevenson LA, Pertwee RG, Henderson A, Toole J, et al. Pharmacological characterization of the anandamide cyclooxygenase metabolite: prostaglandin $\mathrm{E}_{2}$ ethanolamide. J Pharmacol Exp Ther 2002; 301: 900-7.

24. Giufrida A, Parsone LH, Kerr TM, Rodríguez de Fonseca F, Navarro M, Piomelli D. Dopamine activation of endogenous cannabinoid signalling in dorsal striatum. Nat Neurosci 1999; 2: 358-63.

25. Shliker E, Kathmann M. Modulation of transmitter release via presynaptic cannabinoid receptors. Trends Pharmacol Sci 2001; 22: 565-72.

26. Carlisle SJ, Marciano-Cabral F, Staab A, Ludwick C, Cabral GA. Differential expression of the CB2 cannabinoid receptor by rodent macrophages and macrophage-like cells in relation to cell activation. Int Immunopharmacol 2002; 2: 69-82.

27. Walter L, Franklin A, Witting A, Wade C, Xie Y, Kunos K, et al. Nonpsychotropic cannabinoid receptors regulate microglial cell migration. J. Neurosci 2003; 23: 1398-405.

28. Carrier EJ, Kearn CS, Barkmaier AJ, Breese NM, Yang W, Nithipatikom K, et al. Cultured rat microglial cells synthesize the endocannabinoid 2-arachydonyl glycerol, which increases proliferation via a CB2 receptor dependent mechanism. Mol Pharmacol 2004; 60: 155-63.

29. Ramírez BG, Blázquez C, Gómez del Pulgar T, Guzmán M, De Ceballos M. Prevention of Alzheimer's disease pathology by cannabinoids: neuroprotection mediated by blockade of microglial activation. J Neurosci 2005; 25: 1904-13.

30. Waksman Y, Olson JM, Carlisle SJ, Cabral GA. The central cannabinoid receptor (CB1) mediates inhibition of nitric oxide production by rat microglial cells. J Pharmacol Exp Ther 1999; 288: 1357-66.

31. Walter L, Franklin A, Witting A, Möller T, Stella N. Astrocytes in culture produce anandamide and other acylethanolamines. J Biol Chem 2002; 277: 20869-76.

32. Molina-Holgado F, Lledó A. Guaza C. Anandamide suppresses nitric oxide and TNF- $\alpha$ responses to Theiler's virus or endotoxin in astrocytes. NeuroReport 1997; 8: 1929-33.

33. Molina-Holgado F, Molina-Holgado E, Guaza C. The endogenous cannabinoid anandamide potentiates IL- 6 production by astrocytes infected with Theiler's murine encephalomyelitis virus by a receptor-mediated pathway. FEBS Lett 1998; 433: 139-42.

34. Molina-Holgado F, Molina-Holgado E, Guaza C, Rothwell NJ. Role of $\mathrm{CB} 1$ and $\mathrm{CB} 2$ receptors in the inhibitory effects of cannabinoids on lipopolysaccharide induced nitric oxide release in astrocyte cultures. J Neurosci Res 2002; 67: 829-36.

35. Ortega-Gutiérrez S, Molina-Holgado E, Guaza C. Effect of anandamide uptake inhibition in the production of nitric oxide and in the release of cytokines in astrocyte cultures. Glia 2005; 52: 166-8.

36. Molina-Holgado F, Pinteaux E, Moore J, Molina-Holgado E, Guaza C, Gibson RM, et al. Endogenous interleukin-1 receptor antagonist mediates anti-inflammatory and neuroprotective actions of cannabinoids in neurons and glial cells. J Neurosci 2003; 23: 6470-4.

\section{EL SISTEMA CANNABINOIDE EN SITUACIONES DE NEUROINFLAMACIÓN: PERSPECTIVAS TERAPÉUTICAS EN LA ESCLEROSIS MÚLTIPLE}

Resumen. Introducción. El sistema endocannabinoide está constituido por los receptores cannabinoides, los ligandos endógenos y los elementos enzimáticos implicados en su síntesis y degradación. Objetivo. Describir el estado actual de conocimiento sobre la función del sistema como modulador de los procesos neuroinflamatorios asociados con enfermedades crónicas como la esclerosis múltiple. Desarrollo. Los cannabinoides se sintetizan y se liberan en de-
37. Correa F, Mestre L, Docagne F, Guaza C. Activation of cannabinoid $\mathrm{CB} 2$ receptor negatively regulates IL-12p40 synthesis in murine macrophages: role of IL-10 and ERK1/2 kinase signalling. Br J Pharmacol 2005; 145: 441-50.

38. Mestre L, Correa F, Arévalo-Martín A, Molina-Holgado E, Valenti M, Ortar G, et al. Pharmacological modulation of the endocannabinoid system in a viral model of multiple sclerosis J Neurochem 2005; 92 : 1327-39.

39. Zajicek J, Fox P, Sanders H, Wright D, Vickery J, Nunn A, et al, UK MS Research Group. Cannabinoids for treatment of spasticity and other symptoms related to multiple sclerosis (CAMS study): multicentre randomised placeb-control trial. Lancet 2003; 362: 1517-26.

40. Zajicek J, Sansers HP, Wright DE, Vickery PJ, Ingram WM, Reilly SM, et al. Cannabinoids in Multiple Sclerosis (CAMS) study: safety and efficacy data for 12 months follow up. J Neurol Neurosurg Psychiatry $2005 ; 76: 1664-9$.

41. Baker D, Pryce G, Croxford JL, Brown P, Pertwee RG, Huffman JW, et al. Cannabinoids control spasticity and tremor in a multiple sclerosis model. Nature 2000; 404: 84-7.

42. Baker D, Pryce G, Croxford JL, Brown P, Pertwee RG, Makriyannis A, et al. Endocannabinoids control spasticity in a multiple sclerosis model. FASEB J 2000; 15: 300-5.

43. Arévalo-Martín A, Vela JM, Molina-Holgado E, Borrell J, Guaza C. Therapeutic action of cannabinoids in a murine model of multiple sclerosis. J Neurosci 2003; 23: 2511-6.

44. Croxford JL, Miller SD. Immunoregulation of a viral model of multiple sclerosis using the synthetic cannabinoid R (+) WIN55,212. J Clin Invest 2003; 111: 1231-40.

45. Ortega-Gutiérrez S, Molina-Holgado E, Arévalo-Martín A, Viso A, López-Rodríguez ML, Guaza C. Endocannabinoids uptake inhibition as therapeutic approach in a murine model of multiple sclerosis. FASEB J 2005; 19: 1338-44.

46. Cabranes A, Venderova K, De Lago E, Fezza F, Sánchez A, Mestre L, et al. Decreased endocannabinoid levels in the brain and beneficial effects of agents activating cannabinoid and/or vanilloid receptors in a rat model of multiple sclerosis Neurobiol Dis 2005; 20: 207-17.

47. Berrendero F, Sánchez A, Cabranes A, Puerta C, Ramos JA, GarcíaMerino A, et al. Changes in cannabinoid CB1 receptors in striatal and cortical regions of rat with experimental allergic encephalomyelitis, an animal model of multiple sclerosis. Synapse 2001; 41: 195-202.

48. Eljaschewitsch E, Witting A, Mawrin C, Lee T, Schmidt PM, Wolf S, et al. The endogenous anandamide protects neurons during CNS inflammation by induction of MKP-1 in microglial cells. Neuron 2006; 49: 67-79.

49. Trapp BD, Peterson J, Ransohoff RM, Rudick R, Mork S, Bo L. Axonal transection in the lesions of multiple sclerosis. N Engl J Med 1998; 338: 278-85.

50. Pryce G, Ahmed Z, Hankey DJR, Jackson SJ, Croxford JL, Pocok JM, et al. Cannabinoids inhibit neurodegeneration in models of multiple sclerosis. Brain 2003; 126: 2191-202.

51. Nagayama T, Sinor AD, Simon RP, Chen J, Graham SH, Jin K, et al. Cannabinoids and neuroprotection in focal and global cerebral ischemia and neuronal cultures. J Neurosci 1999; 19: 2987-95.

52. Malfait AM, Gallily R, Sumariwalla PF, Malik AS, Andreakos E, Mechoulam R, et al. The nonpsychoactive cannabis constituent cannabidiol is an oral anti-arthritic therapeutic in murine collagen-induced arthritis. Proc Natl Acad Sci USA 2000; 97: 9561-6.

53. Klein TW. Cannabinoid based drugs as anti-inflammatory therapeutics. Nat Rev Immunol 2005; 5: 400-11.

54. Ni X, Geller EB, Eppihimer MJ, Eisenstein TK, Adler MW, Tuma RF. Win 55212,2, a cannabinoid receptor agonist, attenuates leukocyte/ endothelial interactions in a experimental autoimmune encephalomyelitis model. Mult Scler 2004; 10: 258-64.

\section{O SISTEMA CANABINÓIDE EM SITUACÕES DE NEUROINFLAMACÃO: PERSPECTIVAS TERAPÊUTICAS NA ESCLEROSE MÚLTIPLA}

Resumo. Introdução. O sistema endocanabinóide é constituído pelos receptores canabinóides, os ligandos endógenos e os elementos enzimáticos envolvidos na sua síntese e degradação. Objectivo. Descrever o estado actual do conhecimento sobre a função do sistema como modulador dos processos neuroinflamatórios associados a doenças crónicas como a esclerose múltipla. Desenvolvimento. Os canabinóides sintetizam-se e libertam-se a pedido e a 
manda y su producción aumenta en situaciones de neuroinflamación $y$ de daño neural. En este contexto, sus acciones en la microglía y en los astrocitos se caracterizan por una disminución en la expresión de mediadores inflamatorios y de citocinas proinflamatorias. Además, los cannabinoides pueden ejercer acciones neuroprotectoras a través de diferentes tipos de mecanismos y en modelos experimentales de esclerosis múltiple atenúan la sintomatología, disminuyen la inflamación y pueden favorecer la remielinización. Conclusiones. El uso clínico de cannabinoides o agentes farmacológicos que inciden en el sistema endógeno cannabinoide durante la inflamación del sistema nervioso central y en la esclerosis múltiple está actualmente sometido a consideración y debate. El análisis detallado de los resultados obtenidos en la última década ha permitido establecer que son múltiples los mecanismos de actuación de los cannabinoides en patologías del sistema nervioso central que cursan con inflamación crónica y ponen de manifiesto el interés del sistema cannabinoide como nueva herramienta terapéutica. [REV NEUROL 2006; 43: 541-8]

Palabras clave. Cannabinoides. Células gliales. Esclerosis múltiple. Función inmune. Neuroinflamación. sua produção aumenta em situações de neuroinflamação e de lesão neural. Neste contexto, as suas acções na microglia e nos astrocitos caracterizam-se por uma diminuição na expressão de mediadores inflamatórios e de citocinas pró-inflamatórias. Além disso, os canabinóides podem exercer acções neuroprotectoras através de diferentes tipos de mecanismos e em modelos experimentais de esclerose múltipla aliviam a sintomatologia, diminuem a inflamação e podem favorecer a remielinização. Conclusões. $O$ uso clínico de canabinóides ou agentes farmacológicos que incidem no sistema endógeno canabinóide durante a inflamação do sistema nervoso central e na esclerose múltipla é actualmente tido em consideração e submetido a debate. A análise detalhada dos resultados obtidos na última década permitiu estabelecer que são múltiplos os mecanismos de actuação dos canabinóides em patologias do sistema nervoso central que cursam com inflamação crónica e evidenciam o interesse do sistema canabinóide como novo instrumento terapêutico. [REV NEUROL 2006; 43: 541-8]

Palavras chave. Canabinóides. Células gliais. Esclerose múltipla. Função imune. Neuroinflamação. 